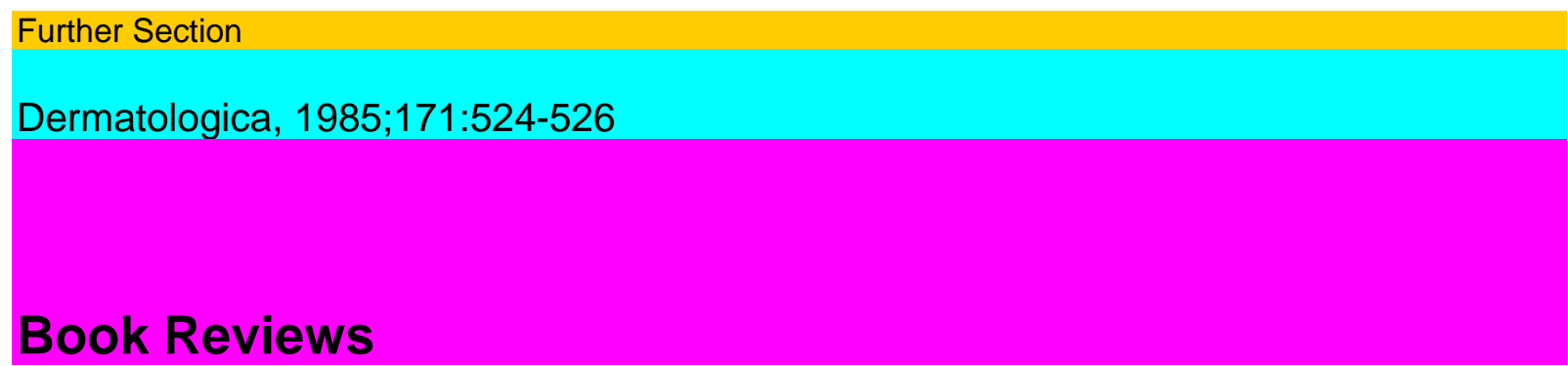

T. Rosen, M.B. Lanning

The Nurse's Atlas of Dermatology

Little Brown, Boston 1983

X + 203 pp.; \$25.75 ISBN 0-316-75705-5

Es ist sicher richtig, dass das Pflegepersonal über Physiologie und Pathologie der Haut instruiert wird. Der Behandlungserfolg allgemein bei Krankheiten wird wesentlich von der Hautpflege beim Patienten und von der Kenntnis allfálliger pathologischer Hautreaktionen bestimmt. Leider wird dieses Ziel im vorliegenden Buch nur zum Teil erreicht, da der entscheidende Teil eines Atlasses, das Bildmaterial, sehr mangelhaft ist. Die Mehrzahl der schwarzweissen Bilder lassen kaum er-kennen, was sie illustrieren sollen, da sie entweder un-genügend kontrastiert oder nicht reliefgetreu aufgenom-men sind. Der Vergleich mit den farbigen Abbildungen zeigt, wie wenig Schwarzweissphotos, vor allem wenn sie nicht auf Kunstdruckpapier reproduziert sind, zur Information des Laien geeignet sind. Dies ist schade, weil der Text, der die Bilder begleitet, sehr informativ ist. Es gibt in kurzer und prägnanter Form die nötigen wis-senschaftlichen Erläuterungen. Sehr brauchbar sind vor allem aber die praktischen Hinweise fur die Pflege, die von Krankenschwestern verfasst sind. Eine Zusammen-stellung der in den USA gebräuchlichen Externa sowie der benötigten Materialien macht das Buch trotz der be-schriebenen Mangel für rein praktische Zwecke nütz-lich.

R. Schuppli, Basel

Siwert Nilsson

Atlas of Airborne Fungal Spores

Springer, Berlin 1983

139 pp.; DM 128.-/USS 55.20

ISBN 3-540-11900-0

Die genaue Kenntnis der 'Airborne Spores' - ge-meint sind mit diesem Ausdruck die durch die Luft ge-tragenen Pilzsporen - ist heute eher eine Sache von Spe-zialisten geworden. Das ist unter anderem darauf zu-rückzuführen, dass die sogenannten Mikrosporen der gleichen Pilzart in ihrem Lebenszyklus in verschiedenen Formen auftreten können. Die lichtmikroskopische Differenzierung wird dadurch in manchen Fallen sehr schwierig.

Mit dem vorliegenden Werk hat der Herausgeber mit seinen fünf Mitarbeitern einem Bedürfnis entsprochen. Sehr eingehend werden in überaus schönen Zeichnun-gen die Lebenszyklen verschiedener Pilze dargestellt. Ebenso werden morphologische Schemata und taxono-mische Besonderheiten erklärt.

Das rasterelektronenmikroskopische Bildmaterial ist vor allem für den Betrachter, der dem Thema etwas ferner steht, sehr informativ und ästhetisch sehr anzie-hend. Die lichtmikroskopischen Bilder hingegen zeigen die grossen Schwierigkeiten, die sich dem Untersucher der täglichen Luftproben auf den Fangstreifen auch mit den allerbesten Lichtmikroskopen stellen. Hier macht sich die Auflösungsgrenze, die beim lichtmikroskopischen Verfahren bei etwa 0,3 $\mu \mathrm{m}$ liegt, bemerkbar. Des-halb können beispielsweise manche sehr häufig 
auftre-tende Sporen, die als kleine Kugeln erscheinen - z.B. Aspergillus und Penicillium -, damit nicht differenziert werden.

Das ganz hervorragend ausgestattete Buch wird dem aufmerksamen Leser, der

lichtmikroskopische und rasterelektronenmikroskopische Bilder vergleicht, in jeder Hinsicht sehr viel bieten können.

Ruth M. Leuschner, Basel

N. Klüken (Hrsg.)

Adipositas, Risikofaktor bei Venen- und

Ly mphgefässerkrankungen?

Ergebnisse der Angiologie, vol. 28 Schattauer, Stuttgart 1983 XI+ 87 pp., 27 fig., 12 tab., kart;

DM 62.-ISBN 3-7945-0931-5

Im vorliegenden zur Schriftenreihe «Ergebnisse der Angiologie» gehörenden 28. Heft werden die Referate zusammengefasst, die 1982 an einem Fortbildungstag der Deutschen Gesellschaft für Phlebologie und Prokto-logie zum Thema der Fettsucht als Risikofaktor bei Venen- und Lymphgefásskrankheiten gehalten wurden. Nach einem einleitenden Referat über die Pathophysio-iogie der Fettsucht, das sehr viel wichtige Information vermittelt, werden in anderen Vorträgen die spezifisch phlebologisch relevanten Fragen behandelt: Das allge-meine Risiko der Adipositas bei Venenkrankheiten, ihre Rolle bei der Entstehung von Lymphödemen, ihr Risiko bei Varizenoperationen, bei der Sklerosierungstherapie und ihr ungünstiger Einfluss auf die Kompressionsthe-

Book Reviews

525

rapie. Ein Kapitel über die Diät bei Ubergewicht leitet zur Diskussion über, an der die Beteiligung offenbar gross war.

R. Schuppli, Basel

Maria Zabel

Zur Histo- und Pathogenese der Nekrobiosis

lipoidica (diabeticorum)

Ergebnisse der Angiologie, vol. 29

Schattauer, Stuttgart 1984

VI+94 pp., 47 fig. (davon 28 mehrfarbig), 2 tab.;

SFr. 62.60

ISBN 3-7945-0963-3

Auch wenn die Necrobiosis lipoidica eine recht sel-tene Hautveränderung ist, so stellt sie für die Erkennung von Stoffwechselanomalien ein wichtiges und aus pa-thophysiologischer Sicht interessantes Krankheitsbild dar. Im vorliegenden Heft werden alle zurzeit bekannten Aspekte zusammengefasst und anhand von 150 fest un-tersuchten Fallen erläutert. Wichtigste Schlussfolge-rung: Die Necrobiosis lipoidica kommt nur in 50\% aller Fälle bei Diabetikern vor, bei alien Patienten lassen sich aber Hinweise auf Gefássveränderungen oder auf eine gestörte Zirkulation finden. Der Text wird durch zum Teil farbige Abbildungen von sehr guter Qualität er-gänzt.

R. Schuppli, Basel

C.S. Potten Radiation and Skin

Taylor \& Francis, London 1985 XI + 225pp.;E21.-ISBN 0-85066-257-5 
Dieses Buch behandelt in 10 Kapiteln die Wechsel-wirkung zwischen Haut und Strahlen, das heisst ioni-sierenden und UV-Strahlen. Im ersten Kapitel wird die Strahlenphysik besprochen. Danach werden die neue-sten Aspekte der Zellstrukturen und Zellteilungsvor-gänge behandelt. Im darauffolgenden Kapitel werden die Strahlenwirkung auf die Zelle sowie deren Reparaturmechanismen besprochen. Normale Struktur und Funktion von Epidermis und Keratin sowie von Dermis und Kollagen werden im 4. Kapitel eingehend darge-stellt. Die Strahleneffekte an Keratoblasten und an der-malem Gewebe sowie solche an den Hautanhangsgebil-den kommen in gesonderten Abschnitten zur Sprache. Die strahlenbedingten Pigmentveränderungen folgen im 8. Kapitel. Der Strahlenreaktion der Langerhans-Zellen ist ein besonderes Kapitel gewidmet. Ein letzter Ab-schnitt enthält allgemeine Bemerkungen über die Strahleneffekte an den verschiedenen Zellsystemen der Haut in Abhängigkeit von der Strahlendosis und derZeit bzw. behandelt die verschiedenen Antwortreaktionen in der Reihenfolge ihres Auftretens nach der Strahleneinwir-kung. Dieses Buch fasziniert in vielfacher Weise: Es ist eine Monographic, ist relativ klein im Format (in etwas knapp über 200 Seiten wird eine Fülle von Material abgehandelt). Wesentliches über Struktur und Funktion von einzelnen Hautabschnitten wird den eigentlichen Strahleneffekten vorangestellt, einige der Abbildungen und Tabellen fassen eine vielseitige Information zusam-men (z.B. Fig. 1.1 und 4.8, Tabellen 3.1 , 4.3,4.4,4.5,7.1 sowie sämtliche Tabellen im letzten Kapitel) und am Schluss eines jeden Themenabschnittes sind einschlä-gige Arbeiten aufgeführt. Allen Interessierten kann dieses Buch wärmstens empfohlen werden.

R. Panizzon, Zurich

F. A. Bahmer

Tropische Hautkrankheiten

Epidemiologie, Immunologie, Diagnostik und

Therapie

Beiträge zur Dermatologie, vol. 10

Hrsg. von /. Metz

Perimed Fachbuch-Verlagsgesellschaft mbH, Erlangen 1984

64 pp., 35 zum Teil farbige Abb.; DM 28-

ISBN 3-88429-167-X

Band 10 der Beiträge zur Dermatologie der Perimed-Serie stellt sich als ein kleines, mit hervorragenden far-bigen Abbildungen illustriertes Heft dar, in dem in aller Kürze einige wichtige, vorwiegend in den Tropen vor-kommende Hautkrankheiten dargestellt sind. Es werden die tiefen Mykosen und die Myzetome behandelt, die Hauttuberkulose, die Lepra und das Rhinosklerom sowie die Leishmaniosen. Alles dies geschieht auf 60 Seiten, wodurch illustriert wird, dass die Beschreibungen der einzelnen Krankheiten sehr knapp gehalten sind. Theoretische Überlegungen fehlen demnach, das Büch-lein ist rein praktischen Gesichtspunkten gewidmet. Dementsprechend kurz sind auch die therapeutischen Anleitungen. Der grosse Vorteil des Büchleins ist, dass es auf persönlichen Erfahrungen des Autors in Mexiko be-ruht, es stellt deshalb ein ideales Orientierungsmittel für den beschäftigten Praktiker dar.

R. Schuppli, Basel

526

Book Reviews

\section{/. Zoltan}


Atlas der Hautersatzverfahren

Karger, Basel 1984

304 pp., SFr. 160.-, DM 192.-, \$ 96.00

ISBN 3-8055-3447-7

Nach dem bereits bekannten Atlas der chirurgischen Schnitt- und Nahttechnik ist dies der zweite grossfor-matige Photo- und Zeichnungsatlas des ungarischen Autors. Der Band kann in einem gewissen Grad als Er-gänzung des obengenannten Werkes gesehen werden. In einem ersten einführenden allgemeinen Teil werden sehr vollständig die Nahlappenplastiken je an einem Beispiel demonstriert. Die komplizierteren Fernlappen vom Arm oder Abdomen werden sehr eingehend be-schrieben, dem Rundstielwanderlappen sind dabei ganze 23 Seiten gewidmet, was uns gegenüber 2 Seiten für musculocutane Lappen doch etwas viel erscheint. Sehr ausführlich wird auch auf die historische Bedeu-tung der einzelnen Verfahren hingewiesen.

Im zweiten Teil wird nun die Anwendung der vor-besprochenen Techniken an verschiedenen Lokalisa-tionen des Körpers mit grossem Schwerpunkt des Ge-sichtes demonstriert. Diese Darstellungen sind, was die konventionellen Lappenplastiken anbelangt, ausseror-dentlich vollständig und instruktiv. Auch für den einge-weihten Chirurgen werden immer wieder neue, kleine Tricks oder technische Einzelheiten klar, an welchen man die grosse Erfahrung des Verfassers erkennt. Die modernen axialen Hautlappenplastiken im Bereich des Rumpfes werden nur kurz gestreift; so fehit etwa für den schon klassischen, oft verwendeten Inguinallappen ein klinisches Beispiel. Ebenfalls vermisst man Beispiele musculocutaner Lappenplastiken, welche doch dank ihrergrossen Sicherheit oft in einzeitigen Operationsver-fahren aufwendige mehrzeitige und risikoreiche kon-ventionelle Lappenplastiken verdrängt haben. Gewisse Körperregionen wie Hüfte, Sitzbein, Perineum werden nicht behandelt. Auch am Unterschenkel sind in der Praxis die angegebenen Lappenplastiken zum grössten Teil durch die moderneren, nur schematisch erwähnten Muskeltranspositionen und musculocutanen Lappen verdrängt worden. 12-20 Zeichnungen und Photogra-phien sind je auf 267 Tafeln zusammengefasst und do-kumentieren Routine- und ausgefallene Probleme der

Defektdeckung in der Plastischen Chirurgie. Die Bild-qualität ist trotz Kleinformats sehr gut und auch für den bereits Erfahrenen aufschlussreich zusammengestellt. Das Buch ist für historisch interessierte plastische Chirurgen als Einführung in die allgemeine Technik der Lappenplastiken und für die in der Praxis oft so wich-tigen Nahlappenplastiken hervorragend und instruktiv. Als Ratgeber für ausgedehnte Defektdeckungen ist das Werk wegen Fehlen von Beispielen der modernen Mus-kel- und musculocutanen Lappen sowohl gestielt wie frei nicht zu empfehlen. Dem Buch folgt ein ausführ-liches Literaturverzeichnis von über 1000 Referenzen, welches trotz des östlichen Ursprungs des Buches jedoch (leider) zu fast 98\% allgemein zugängliche westliche Pu-blikationen enthält.

N. Lüscher, Basel

P.F. Meredith

Les brûlures thermiques de la main

Traitement chirurgical Médecine et Hygiene, Geneve 198484 pp., Fr. 30.-ISBN 2-88049-016-2 Obwohl der Dermatologe seiten Gelegenheit hat und in den Fall kommt, frische Verbrennungen und Verbrü-hungen der Hand behandeln zu müssen, ist doch die Kenntnis der Möglichkeiten der operativen Korrektur von Verbrennungsfolgen für ihn sehr nützlich. Er hat ja hie und da Keloidbildungen als Verbrennungsfolge an den Händen zu beurteilen. In dem kleinen Büchlein sind nach allgemeinen Kapiteln über Verbrennungsfolgen und Verbrennungsursachen die operativen Möglichkeiten geschildert, die heute zur Behandlung der wichtig-sten 
Verbrennungsfolgen, der narbigen Kontrakturen, bestehen. Weiter werden die Verbandtechnik zur Verhü-tung von Keloiden und von hypertrophischen Narben sowie die physikalischen und ergotherapeutischen Massnahmen dargestellt. Zahlreiche schematische Zeichnungen, Tabellen und Photographien ergänzen den Text.

R. Schuppli, Basel 